\title{
PALATAL VERSUS BUCCAL ANTRAL APPROACH FOR MAXILLARY SINUS LIFTING AND IMPLANT PLACEMENT
}

\author{
Omar N. EL-prince ${ }^{1} M D$, Abdelaziz F. Khalil ${ }^{2} P h D$, Ahmed M. EL-sabbagh ${ }^{2} P h D$, Magued H. \\ Fahmy ${ }^{2} P h D$
}

\section{ABSTRACT}

INTRODUCTION: Implant placement in the maxillary posterior alveolar ridge is often complicated by post extraction bone resorption, pneumatization of maxillary sinuses, and poor quality of alveolar bone. In these situations, the residual vertical bone height is reduced making standard implant placement difficult. Elevation of the maxillary sinus floor using the lateral antral approach is one possible solution. Palatal window osteotomy approach for maxillary sinus floor lifting is a new surgical technique used to increase vertical bone height prior to implant placement.

OBJECTIVES: This study was designed for clinical evaluation of the maxillary sinus lifting technique using a palatal approach versus buccal approach.

MATERIALS AND METHODS: this study was carried out as a randomized controlled clinical trial, the study sample included 14 patients. The sample was selected conveniently to fulfill a list of inclusion and exclusion criteria. All patients were divided into two equal groups, had gone maxillary sinus lifting together with implant placement, group1 :( study group) seven patients had sinus lifting through the palatal approach technique simultaneously with implant insertion. In group2 (control group) seven patients had maxillary sinus lifting using buccal antral approach technique simultaneously with implant insertion. Clinical and radiographic evaluation was done through 6 months postoperatively.

RESULTS: Regarding postoperative clinical evaluation, group 1 was superior to that of group 2 in tissue management, because the vestibular anatomy in this group was not altered neither postoperative swelling occurred consequently nor disharmonious soft tissue scarring. Regarding the postoperative radiographic evaluation, group 1 was significantly increased in bone density around dental implants and less marginal bone loss postoperatively, however group 2 had higher vertical amount of bone gained around implants postoperatively.

CONCLUSIONS: The palatal sinus lifting approach permitted higher postoperative comfort, less postoperative edema, less marginal bone loss around implants, and higher bone density around implants postoperatively.

KEYWORDS: Palatal sinus lifting approach, buccal sinus lifting approach, dental implants.

RUNNING TITLE: Palatal window osteotomy for maxillary sinus lifting.

1-MD Oral and Maxillofacial Surgery, Faculty of Dentistry, Alexandria University, Egypt.

2-Professor of Oral and Maxillofacial Surgery, Faculty of Dentistry, Alexandria University, Egypt.

\section{INTRODUCTION}

Replacement of missing teeth has become one of the most important needs for patients attending clinics to restore esthetics and function. Many treatment modalities are available for replacing a single missing tooth; removable partial denture, fixed partial denture or dental implant. Each modality is a possible treatment option and has its own advantages and disadvantages (1).

Implant treatment is an excellent alternative to replace a missing tooth. Brånemark et al. (2) in 1974 was the first to describe the bone to implant contact, called osseointegration. Albrektsson et al. (3) defined the term at the light microscope level "direct contact between living bone and implant”.

Dental implantology at the beginning of the Third Millennium can replace tooth defects almost always if they occur in an adult individual who is willing to cooperate and to provide a financial contribution. Contraindications for implants are increasingly being reduced. Thanks to the augmentation procedure, we know of virtually no situations that the implant could not be implanted due to insufficient quantity of the alveolar bone (4).

Loss of teeth will in itself result in reduced bone volume as well as trauma from removable dentures or from different pathologies (5).

Implant placement is often complicated in the posterior upper jaw by post extraction bone resorption, pneumatization of maxillary sinuses, and poor quality of alveolar bone. In these situations, the residual vertical bone height is reduced making standard implant placement difficult (6). The elevation of the maxillary sinus floor is one possible solution (7).

Boyne (8) in 1980 was the first to introduce maxillary sinus floor augmentation with autologous bone graft. This technique has been modified and improved by Tatum in 1986 (9) who introduced the lateral approach by fenestrating the buccal wall of maxillary sinus and lifting the Schneiderian membrane. This technique was modified by Wood and More (10) in 1994 where they grafted the maxillary sinus with intra-orally harvested autogenous bone in order to place implant in the edentulous jaw.

Currently, two main approaches to the maxillary sinus floor elevation procedure can be found in the literature. The first approach, lateral antrostomy, is the classic and the more commonly performed technique originally described by Tatum. Later, Summers (11) advocated a second approach: the crestal approach, using osteotomes.

Lateral antrostomy is started with a crestal incision made on the alveolar ridge. A full-thickness flap is then raised to allow access to the lateral antral wall. Once the flap has been raised to a desired level, antrostomy is performed to create a trapdoor on the lateral buttress of the maxilla. The sinus membrane is then gently lifted from the bony floor by means of an antral curette (12). It is important to free up the sinus membrane in all directions before attempting to intrude the trapdoor medially. A space is 
created after the sinus membrane has been elevated by the intruded trapdoor. This space is then grafted with different materials to provide the platform for implant placement $(13,14)$.

Implants are placed either simultaneously with the graft (1-stage lateral antrostomy) or after a delayed period of up to 12 months to allow for graft maturation (2-stage lateral antrostomy) (15).

One of the drawbacks of the lateral antrostomy is that it requires the raising of a large flap for surgical access. Summers proposed a conservative crestal approach using osteotomes for maxillary sinus floor elevation in 1994 (11).

A crestal approach for sinus floor elevation was initially suggested by Tatum (9). Summers (11) later proposed the osteotome technique to place implants in a simpler, more conservative, and less invasive manner than the lateral approach (16). In Summers' technique, an osteotome is inserted through the edentulous alveolar crest at the inferior border of the maxillary sinus floor. This intrusion procedure produces a fracture in the least traumatic way possible and the sinus floor is moved upward. This creates a space for bone graft and simultaneous implant placement (17-19).

Based on these two principal concepts for sinus augmentation, other different and modified techniques for the crestal and lateral approach to the maxillary sinus have been introduced by Stübinger et al (20) in 2010 when he introduced a new palatal approach to the maxillary sinus using piezo-surgery for maxillary sinus elevation. Stübinger showed the main advantages of this new technique as favorable soft tissue management, and valuable aggregation and condensation of the applied augmentation material at the inner palatal part of the alveolar crest.

The aim of the current study was to evaluate the maxillary sinus lifting technique using a palatal approach versus buccal approach.

\section{MATERIALS AND METHODS}

\section{Informed Consent:}

Appropriate institutional ethical clearance from the Faculty Ethical Committee and written informed consent from the patients were obtained. All patients were informed about the aim of the study.

\section{Study Design:}

This study was carried out as a randomized controlled clinical trial.

\section{Patient Selection and Evaluation:}

In this study 14 patients requiring dental implants rehabilitation in the maxillary posterior region, where the alveolar ridge was severely resorbed and elevation of maxillary sinus floor was indicated were selected from the Outpatient Clinic of Oral and Maxillofacial Surgery Department, Faculty of Dentistry, Alexandria University.

The inclusion criteria of this study were: Patients had maxillary posterior unilateral or bilateral free end saddle, Bone height in relation to maxillary sinus floor was less than $7 \mathrm{~mm}$, Patients ages ranged from 30 to 60 years old, patients had acceptable inter-arch space for the future prosthesis, and patients had adequate oral hygiene. The exclusion criteria were: patients with chronic sinusitis or sinus pathologies, heavy smoking and/or alcoholism patients, patients with para-functional habits, any systemic disease directly affecting upon surgical procedure and/or bone healing, patients on medications that may affect the procedure and psychiatric patients.
The selected patients were allocated randomly in two equal groups: Group 1: (study group) which included seven patients, where maxillary sinus lifting was performed using a palatal window osteotomy technique together with implant placement and Group 2: (control group) which included seven patients, where maxillary sinus lifting was performed using a buccal antral approach technique together with implant placement.

\section{Materials}

- Superline implants and Surgical kit for implant placement (Dentium Co., Ltd. Seoul, korya)

Piezo- ultrasonic surgery unit piezotome 2 (Acteon Co., UK)

-Dentium Advanced Sinus Kit DASK (Dentium Co., Ltd. Seoul, korya) which consists of lateral and crestal approach drills, and it also contains a set of sinus elevators instruments.

\section{Pre-surgical phase:}

All patients were evaluated by proper case history and thorough clinical and radiographic examination using Cone beam computed tomography CBCT (J.morita, at $8 \mathrm{~mA}, 90$ $\mathrm{KV}$, Japan) and OnDemand3D ${ }^{\mathrm{TM}}$ software program (OnDemand3DTM Goddard Way, Suite 250 Irvine, CA 92618 USA) to decide the indication for sinus lifting through either a palatal or buccal window osteotomy approach and to avoid the presence of vital structure that may interfere with the osteotomy site (Figure 1).

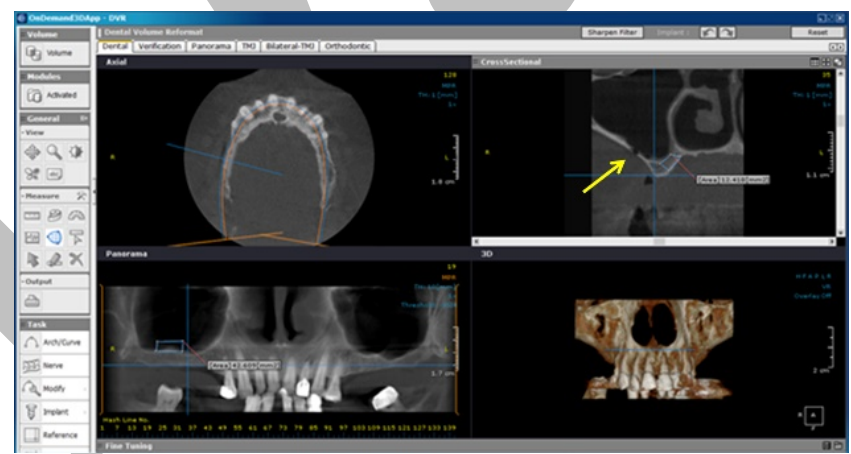

Figure (1):Properative CBCT for virtual planning of platal approach technique in right side and buccal approach technique in left side,with the Posterior Superior Alveolar Artery (PSSA) evident in coronal view in right side (arrow).

ENT consultation was made to exclude any inflammatory or pathological condition related to the maxillary sinus before the operation.

Maxillary and mandibular full arch Impressions were taken and a diagnostic wax up was performed on the study cast to fabricate a vacuum-formed stent to locate the osteotomy site during surgery and the site of implant location.

\section{Surgical Procedure:}

All patients in both groups were operated under general anesthesia with intubation and strict aseptic conditions.

In group 1: A full-thickness palatal flap was raised after a slightly palatally located crestal and beveled incision extending from the incisive papilla area till between the area of upper first and second molar to access the palatal bony wall of the maxillary sinus. The flap was carefully held off with fixation sutures. The palatal window osteotomy was performed using piezosurgery device (Piezotome 2, Acteon Co., UK) guided by the study cast and the virtual pre 
planning by CBCT. Then a complete window osteotomy for palatal sinus elevation was carried out with a rectangular pattern which varied in size and position in each case depending on the presurgical virtual planning (Figure 2 a \&b). After osteotomy, the palatal bony lid was completely removed and preserved in saline solution (Figure $2 \mathrm{c}$ ). The sinus membrane was meticulously dissected and lifted and then the sinus lifting elevator was glided along the bone in three directions of the palatal window, anterior, posterior and superior to ensure the release of the membrane (without tearing) from the sharp bony access margins. Drilling for implant placement was done using Dentium implant system (Dentium Co., Ltd. Seoul, korya) guided by the preformed surgical stent, And Implant of appropriate size was initially engaged at the apical aspect of the alveolar ridge osteotomy to maintain the Schneiderian membrane in the desired elevated position around the implant (tenting) (Figure $2 \mathrm{~d}$ ). Then the palatal window osteotomy was repositioned into its former place. Flap was then repositioned and closed with Vicryl 4/0 (Johnson \&Johnson, Co, Egypt.) horizontal mattress suture.

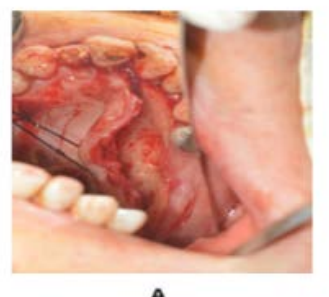

A

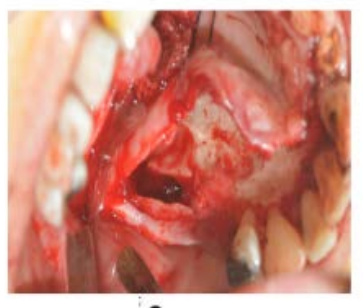

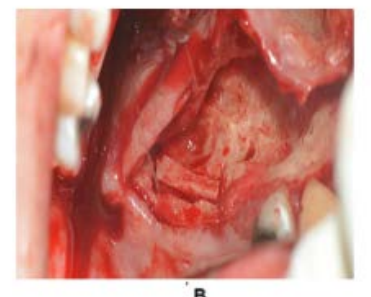

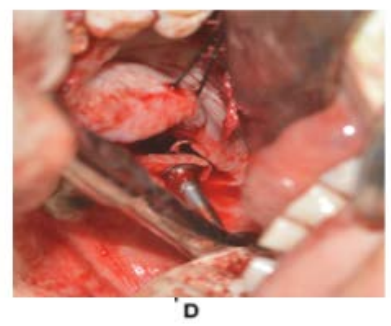

Figure (2): A photograph showing (A) raising of the palatal
flap, (B) Piezoelectric palatal window osteotomy, (C) intact flap, (B) Piezoelectric palatal window osteotomy, (C) intact
schniderian membrane after palatal window osteotomy removal and (D) implant insertion after sinus lift.

In group 2 (control group): A mid-crestal or a slightly palatal incision was performed, leaving at least three mm of attached gingiva on the facial aspect of the incision with anterior and posterior vertical releasing incisions. These vertical incisions should be at least $5 \mathrm{~mm}$ away from the planned osteotomy then a full-thickness mucoperiosteal flap was raised and the lateral aspect of the maxillary sinus was exposed. The buccal window osteotomy was performed at the premolars -molar area using the piezo-surgery device (Figure 3 a, b \& c) and the sinus membrane was meticulously dissected and lifted using Sinus membrane periosteal elevators (Dentium Advanced Sinus Lift Set). The elevator was glided along the bone in three sides of the window, anterior, inferior and posterior to ensure release of the membrane (without tearing) from the sharp bony access margins. Drilling for implant placement was done guided by the preformed surgical stent. Implant of appropriate size was initially engaged at the apical aspect of the alveolar ridge osteotomy to maintain the Schneiderian membrane in the desired elevated position around the implant (tenting)
(Figure $3 \mathrm{~d}$ ). Then the buccal window osteotomy was repositioned into its former place. Finally the flap repositioned and closed with sutures.
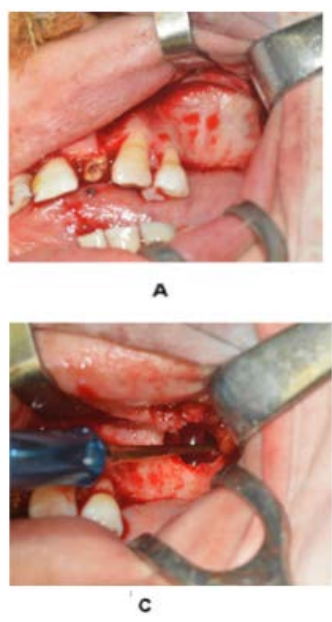
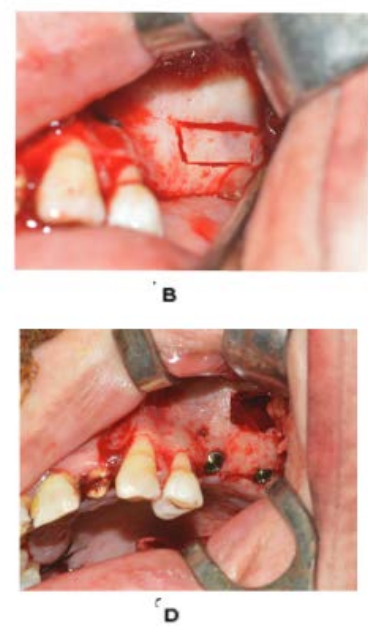

Figure (3): A photograph showing (A) Preoperative view of buccal left side, (B) Piezoelectric buccal window osteotomy for left side, (C) elevation of the schniderian membrane and (D) after implant insertion.

Post-surgical instructions and medications:

All patients were advised to: apply Cold fomentation over the cheek at five minutes interval for one hour on the first day, and to avoid sneezing, nose blowing or other actions that might create high intranasal pressure or vacuum. Patients also were instructed to avoid drinking with straws for a week. Patients were instructed not to wear any prosthesis over the surgical site for at least one week after surgery, and a soft high nutrient diet was recommended.

Postoperative medication included broad spectrum antibiotic Amoxicillin $875 \mathrm{mg}$ + Clavulanic acid $125 \mathrm{mg}$ tablets (Augmentin 1 gram tablet GlaxoSmithKline group. Co. Egypt) every 12 hours for five days to avoid postoperative infection. Non-steroidal anti-inflammatory analgesic (Brufen 600mg tablets Abbott house. Co. Egypt) every 8 hours for three days to avoid the possibility of pain. Nasal Decongestant as Oxymetazoline (Afrin 0.05 nasal drops Schering plough Co. Egypt) every 8 hours for five days.

\section{Postoperative evaluation:}

All patients were examined the day after surgery then weekly for the first month postoperatively, then on intervals of one, three and six months postoperatively. Pain and discomfort were examined using visual analogue scale (VAS) (21). Edema was evaluated by a visual descriptor analogue scale (22) was used to indicate presence/absence of edema and inflammation. Wound healing was evaluated by Early Wound Healing Index (EHI) (23) to provide a more objective assessment of postoperative wound healing quality.

Immediate Postoperative and 6 months postoperative CBCT scan were conducted with the same apparatus and settings as the preoperative scans to evaluate the amount of vertical bone height gained, amount of bone density, and amount of marginal bone loss postoperatively (Figure 5). 


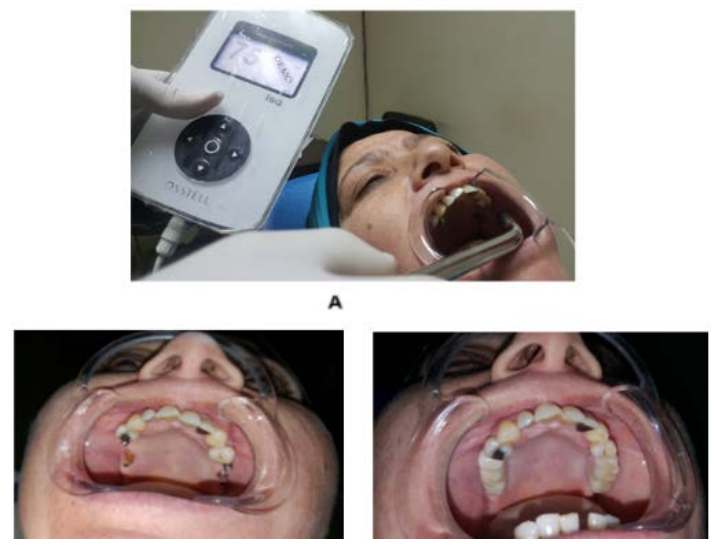

Figure (4): A photograph showing (A) checking implant stability before abutment insetion using osstell, (B) after abutment insertion and $(\mathrm{C})$ porcelain fused to metal crowns delivered to patient.

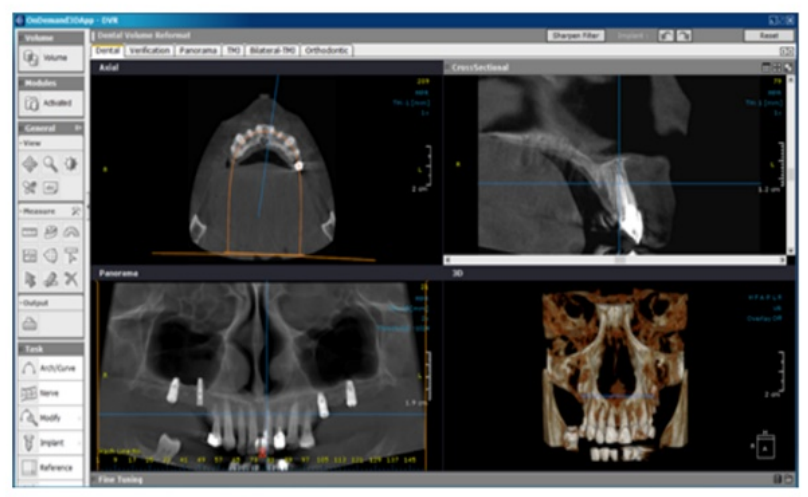

Figure (5): 6 months postoperative cone beam CBCT (palatal approach in right side and buccal approach in left side).

For evaluation of the amount of bone density, the data from CBCT was recorded in Hounsfield unit (HU) and comparison was made between preoperative (base line), immediate postoperative and 6 months postoperatively.

The data from CBCT also was recorded in millimeter (mm) and comparison was made between preoperative, immediate postoperative and 6 months postoperatively for evaluation of vertical bone height gained.

To assess the amount of marginal bone loss postoperatively, data from CBCT also was recorded in millimeter $(\mathrm{mm})$ and comparison was made between immediate postoperative and 6 months postoperatively.

\section{Prosthetic phase:}

All implants stability were checked after 6 months postoperatively using Osstell (Osstell Co. Sweden) implant stability meter before prosthetic loading. (Figure 4 a). Reopening of the implant side was performed by punch technique in both groups. Gingival formers was inserted for 1-2 weeks to provide good gingival contour around implant collar. Impression was taken using implant analogues. Definitive porcelain fused to metal crowns were delivered to all patients after 6 months for both groups (Figure 4 b \& c).

\section{Statistical analysis of the data (24)}

Data were fed to the computer and analyzed using IBM SPSS software (Package version 20.0. IBM Corporation, 1
New Orchard Road, Armonk, New York, United States). Quantitative data were described using range (minimum and maximum), mean, standard deviation and median. The distributions of quantitative variables were tested for normality using Kolmogorov-Smirnov test, Shapiro-Wilk test and D'Agstino test. If it reveals normal data distribution, parametric tests were applied. If the data were abnormally distributed, non-parametric tests were used. For abnormally distributed data, comparison between two independent populations was done using Mann Whitney. Significance of the obtained results was judged at the $5 \%$ level.

\section{RESULTS}

In this study, seven patients in group 1 had maxillary sinus lifting from palatal approach simultaneously with implant placement and in group 2 seven patients had maxillary sinus lifting from buccal approach simultaneously with implant placement. The selected patients were nine males (64\% of patients) and five females (36\% of patients), and their age ranged from 33-59 years with a mean age of 44.5 years.

In group 1 the mean height of the alveolar ridge from the marginal crest to floor of the maxillary sinus was $5.36 \pm$ $1.17 \mathrm{~mm}$ (Range: $2.90-7.0 \mathrm{~mm}$ ), while in group 2 mean height of the alveolar ridge from the marginal crest to floor of the maxillary sinus was $5.28 \pm 1.21 \mathrm{~mm}$ (Range: $3.20-$ $7.0 \mathrm{~mm})$,.

Regarding the number of implants placed, seventeen implants were placed in group 1 patients and fifteen implants were placed in group 2 patients.

Regarding the distributions of implants by position, in group 1, five implants were placed in upper second molar area, nine implants were placed in upper first molar region and three implants in upper second premolar area. In group 2, four implants were place in upper second molar region, eight implants in upper first molar region, and two implants in upper second premolar area.

\section{Post-operative evaluation:}

\section{Clinical evaluation}

Regarding postoperative pain evaluation in group 1 five patients experienced mild pain $(\mathrm{VAS}=1$ ) and two patients experienced moderate pain (VAS $=2$ ) at surgical site for 1-5 days duration. In group 2 three patients experienced moderate pain $(\mathrm{VAS}=3)$ and four patients experienced moderate to severe pain (VAS $=4-7$ ) at surgical site for 1-5 days duration.

Regarding post-operative edema in group 1 four patients experienced no swelling grade 0 edema and three patients experienced slight swelling grade 1 edema that lasted for five days and then the edema subsided gradually. However in group 2 two patients had experienced a slight swelling grade 1 edema, three patients had experienced a mild swelling grade 2 edema, and two patients had experienced a severe swelling grade 3 edema, that lasted for five days and then the edema subsided gradually.

For evaluation of postoperative surgical wound healing in group 1 five patients had experienced grade 1 Early Wound Healing Index (EHI) and two patients had experienced grade 2 Early Wound Healing Index (EHI). However in group 2 four patients had experienced grade 2 Early Wound Healing Index (EHI) and two patients had experienced grade 1 Early Wound Healing Index (EHI) and one patient had experienced grade 3 Early Wound Healing Index (EHI). 
Implant stability was checked for all implants using osstell (Osstell Co. Sweden) 6 months postoperatively. In group 1 the minimum ISQ number recorded was 61 and maximum recorded ISQ number was 79. However in group 2 the minimum ISQ number recorded was 64 and maximum recorded ISQ number was 77.

\section{Radiographic evaluation}

The amount of bone density was evaluated by comparing the preoperative, the immediate postoperative and 6 months postoperative CBCT images for all thirty two implants in both groups. The amount of bone density of newly formed bone in group 1 was significantly higher than that of group 2 after six months postoperatively (Table 1 ).

The amount of vertical bone height gained was evaluated by subtracting the difference between immediate postoperative bone height gained and six months postoperatively. The amount of vertical bone height gained in group 2 was significantly higher than that in group 1 .

Data were also collected for each implant regarding the amount of marginal bone loss by subtracting the difference between the marginal bone level in the immediate postoperative phase and six months later. The amount of marginal bone loss after six month in group 2 was highly significant than that of group 1 (Table 2).

Table (1): Comparison between the two groups according to bone density of the newly formed bone in Hounsfield unit (HU)

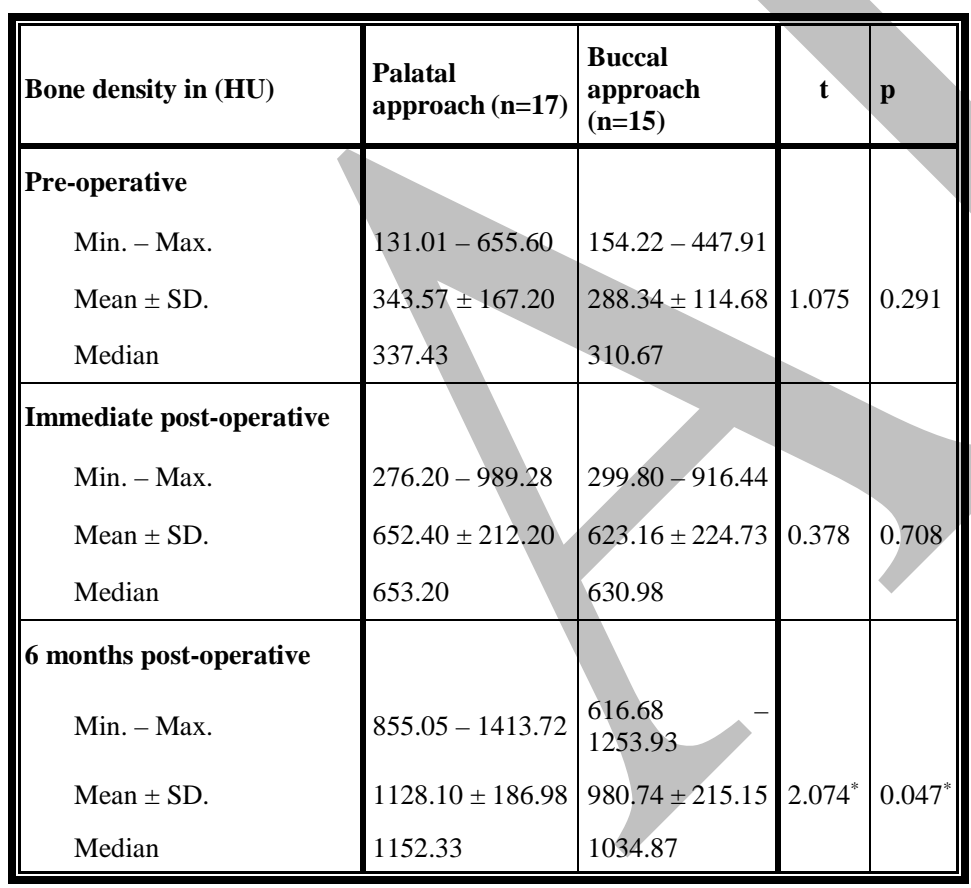

$\mathrm{t}, \mathrm{p}$ : $\mathrm{t}$ and $\mathrm{p}$ values for Student t-test for comparing between the two groups

*: Statistically significant at $\mathrm{p} \leq 0.05$
Table (2): Comparison between the two groups according to marginal bone loss after 6 month

\begin{tabular}{|c|l|l|l|l|}
\hline $\begin{array}{l}\text { Marginal bone loss after } \\
\text { 6 month }\end{array}$ & $\begin{array}{l}\text { Palatal } \\
\text { approach } \\
(\mathbf{n = 1 7 )}\end{array}$ & $\begin{array}{l}\text { Buccal } \\
\text { approach } \\
(\mathbf{n = 1 5 )}\end{array}$ & $\mathbf{t}$ & $\mathbf{p}$ \\
\hline $\begin{array}{c}\text { Min. - Max. } \\
\text { Mean } \pm \text { SD. }\end{array}$ & $\begin{array}{l}0.40-1.72 \\
\text { Median }\end{array}$ & $0.50-4.73$ & & \\
\hline
\end{tabular}

$\mathrm{t}$, p: $\mathrm{t}$ and $\mathrm{p}$ values for Student t-test for comparing between the two groups

*: Statistically significant at $\mathrm{p} \leq 0.05$

\section{DISCUSSION}

Implant placement is often complicated in the posterior segment of the upper jaw by post extraction bone resorption, pneumatization of maxillary sinuses, and poor quality of alveolar bone. In these situations, the residual vertical bone height is reduced making standard implant placement difficult (6). Elevation of the maxillary sinus floor is one possible solution (7).

In this study; evaluation of the maxillary sinus floor elevation technique using a palatal antral approach versus buccal antral approach has been accomplished by selecting 14 patients, they were divided into two equal groups for comparison between the two techniques clinically and radiographically.

Virtual planning by CBCT was made to decide the indication for sinus lifting through either a palatal or buccal window osteotomy approach to avoid the presence of vital structure that may interfere with the osteotomy site like Posterior Superior Alveolar artery (PSAA) which can be visualized in coronal view as mentioned by Hayek E et al (25) in 2015. This was in accordance with one case in the present study, where there was PSAA at the buccal aspect in CBCT, and guided the operator to include the patient in group1.

As stated by Stübinger et al (20) in 2010 the residual shape of the palatal vault is an additional anatomic aspect that has to be considered when choosing between the buccal and palatal approach technique for sinus lifting. In cases of a very narrow vault, not only the view of the operation site will be reduced but also the surgical access for the palatal approach will be difficult. Therefore in such cases the buccal approach was chosen in the present study.

In this study the minimum alveolar ridge height below the maxillary sinus floor before surgery was $2.9 \mathrm{~mm}$ with an average of bone height in group 1 was $5.36 \pm 1.17$ and in group 2 was $5.28 \pm 1.21$ that achieved a satisfied primary stability to the inserted implant. This was in agreement with Nedir et al (26) in 2006 who confirmed that the minimal requirement for achieving primary stability is the presence of a cortical bone layer. This was also in accordance with He et al (27) in 2011 who showed that it was possible to achieve implant stability even when the available bone height was limited down to 4-5 $\mathrm{mm}$.

Regarding the window osteotomy to access the maxillary sinus area in both groups, piezoelectric osteotomy and removal of the osseous lid was performed. By very thin 
piezoelectric osteotomy lines with minimal loss of bone volume, the lid could be perfectly repositioned into the recipient site with only a little residual gap in group1 considering that the palatal bone is thick enough for this manipulation compared with the buccal bone. The favorable healing with complete and undisturbed incorporation of the bone window into the alveolar crest makes this method a promising alternative to the top hinge door technique which done buccally. This method of bony window repositioned came in agreement with Sohn et al. (28) in 2011 who reported that the repositioned bony window may accelerate new bone formation earlier during healing versus placement of a collagen membrane grafting material over the site of the window opening.

Regarding the postoperative clinical evaluation; in this study it was found that patients in group1 with palatal approach had superior wound healing and less pain and edema compared to that in group 2 with buccal approach, this results came in the same line with what described by Stübinger et al (20) in 2010, who explained the superiority of palatal flap in postoperative healing was due to the absence of any muscular traction forces or functional movements on the palatal side compared with the buccal vestibular side.

Despite the fact that all implants in this study were placed simultaneously with maxillary sinus membrane lifting without adding any grafted material, bone formation was evidenced and recorded around all implants at six months postoperatively in both groups. At six months the increased bone density was found to be statistically significant (p-value $<0.001)$. This result emphasized the fact that the Schneiderian membrane itself has an osteogenic effect and came in accordance with the same result stated by Hatano et al (29) in 2007, Sani et al (30) in 2008 and Balleri et al (31) in 2012.

Regarding the comparison between the two groups about the amount of newly bone formation postoperatively it was found in the present study that group1 had a significant higher newly amount of bone formation after 6 months postoperatively than that of group 2. This can be theoretically explained due to the fact that in group 1 there was preservation of the blood supply and nutrients to the implant sinus interface area while blood supply may be compromised in group 2 where there is severing the blood supply by raising the buccal flap, this came to an agreement with Stübinger et al (20) who compared between the blood supply of the two mentioned approaches and found that raising the buccal flap could compromise the underneath thin buccal bone blood supply from the periosteam.

The amount of vertical bone height gained after 6 months postoperatively was significantly higher in group 2 than in group 1 . This can be explained duo to the fact that palatal approach still had a limited accessibility for sinus lifting comparing to the buccal approach due to the narrower area of window osteotomy in the palatal vault than that window osteotomy done along the surface of the buccal area which allow us to lift the schniderian membrane more superiorly simultaneously with insertion of longer implants in group 2 with buccal approach. This came to an agreement with Stübinger et al (20) who explained the difficulty of the palatal approach was due to narrow vault that will reduce the surgical access to the maxillary sinus.

Regarding the marginal bone loss (MBL) after six months postoperatively compared with that level at the immediate postoperative period, it was found that there was a significant lower MBL in group 1 than in group 2. This can be explained by disturbance to the periosteum during reflection and reposition of the buccal flap compared to the perfectly fitted and less blood supply disturbance of the palatal flap, this was in agreement with Stübinger et al (20) in 2010 who explained that as the resorption of the cancellous bone inside the alveolar crest progresses, the lateral and inferior walls of the sinus are reduced to paper thin lamellae of cortical bone, which were no longer supplied by intraosseous vessels, but solely by the vessels in the local periosteum. The preservation of the periosteal blood supply at the buccal side of the lateral side of the maxilla is therefore of special importance for protecting the bone from additional resorption.

\section{CONCLUSIONS}

We concluded from this study that the palatal sinus lifting approach permitted higher postoperative comfort, less marginal bone loss around implants postoperatively, and higher bone density around implants postoperatively. This technique presents an attractive alternative in cases where enough width and height of the posterior alveolar crest is available.

\section{CONFLICT OF INTEREST}

The authors declare that they have no conflicts of interest.

\section{REFERENCES}

1. Christensen GJ. Elective vs. mandatory dentistry. J Am Dent Assoc. 2000;131:1496-8.

2. Branemark AI, Hansson BO, Adell R, Breine U, Lindstrom J, Hallen O, et al. Osseointegrated implants in the treatment of the orthognathic treated jaw. Experience from a 10-year period. Scand J Plast Reconstr Surg Suppl. 1974;16:3-24.

3. Albrektsson T, Johansson C. Osteoinduction, osteoconduction and osseointegration. Eur Spine J. 2003;2:12-22.

4. Jensen OT. The sinus bone graft. Chicago, London: Quintessence Publ.; 1999. p. 52

5. Chanavaz M. Maxillary sinus dextra sinistra: anatomy, physiology, surgery, and bone grafting related to extraction--eleven years of surgical experience (19791990). J Oral Implantol. 1992;12:192-200.

6. Misch CE, Steignga J, Barboza E, Misch-Dietsh F, Cianciola LJ, Kazor C.. Short dental implants in posterior partial edentulism: A multicenter retrospective 6-year case series study. J Periodontal. 2006;77:1340-7.

7. Del Fabbro M, Rosano G, Taschieri S. Implant survival rates after maxillary sinus augmentation. Eur J Oral Sci. 2008;116:497-506.

8. Boyne PJ. Grafting of maxillary sinus floor with autogenous marrow and bone. J Oral Surg. 1980;38:6136.

9. Tatum Jr H. Maxillary and sinus implant reconstructions. Dent Clin North Am. 1986;30:207-29.

10. Wood RM, Moore DL. Grafting of the maxillary sinus with intraorally harvested autogenous bone in order to place implant in the edentolous jaw. Int J Oral Maxillofac Implants. 1994;3:201-12. 
11. Summers RB. A new concept in maxillary implant surgery: the osteotome technique. Compend Contin Educ Dent. 1994;15:152-62.

12. Marx RE, Garg AK. A novel aid to elevation of the sinus membrane for the sinus lift procedure. Implant Dent. 2002;11:268-71.

13. Raghoebar GM, Timmenga NM, Reintsema $H$, Stegenga B, Vissink A. Maxillary bone grafting for insertion of endosseous implants: results after 12-124 months. Clin Oral Implants Res. 2001;12:279-86.

14. Kahnberg KE, Ekestubbe A, Gröndahl K, Nilsson P, Hirsch JM. Sinus lifting procedure. I. One-stage surgery with bone transplant and implants. Clin Oral Implants Res. 2001;12:479-87.

15. Zitzmann NU, Scharer P. Sinus elevation procedures in the resorbed posterior maxilla: Comparison of the crestal and lateral approaches. Oral Surg Oral Med Oral Pathol Oral Radiol Endod. 1998;85:8-17.

16. Summers RB. The osteotome technique: Part 3. Less invasive methods of elevating sinus floor. Compend Contin Educ Dent. 1994;15:698-708.

17. Cosci F, Luccioli M. A new sinus lift technique in conjunction with placement of 265 implants: A 6-year retrospective study. Implant Dent. 2000;9:363-8.

18. Fugazzotto PA. The modified trephine/osteotome sinus augmentation technique: Technical considerations and discussion of indications. Implant Dent. 2001;10:25964.

19. Ping-Yuen F. Piezoelectric-assisted osteotomemediated sinus floor elevation: An innovative approach. Implant Dent. 2010;19:299-305.

20. Stübinger S, Saldamli B, Landes CA, Sader R. Palatal piezosurgical window osteotomy for maxillary sinus augmentation. Int $\mathrm{J}$ Oral Maxillofac Surg. 2010;39:606-9.

21. Chang DJ, Bird SR, Bohidar NR, King T. Analgesic efficacy of rofecoxib compared with codeine/acetaminophen using a model of acute dental pain. Oral Surg Oral Med Oral Pathol Oral Radiol Endod. 2005;100:e74-80.

22. Henrikson PA, Thilander H, Wåhlander LA. Voltaren as an analgesic after surgical removal of a lower wisdom tooth. Int J Oral Maxillofac Surg.1985;14:3338.

23. Wachtel H, Schenk G, Bohm S. Weng D, Zuhr O, Hurzeler M. Micro•surgical access flap and enamel matrix derivative for the treatmentof periodontal intrabony defects: A controlled clinical study. J Clin Periodontal. 2003;30:496-504.

24. Kotz S BN, Read CB, Vidakovic B. Encyclopedia of statistical sciences. N.J.: Wiley-Interscience; 2006. pp 33-59.

25. Hayek E, Nasseh I, Hadchiti W, Bouchard P, Moarbes M, Khawam G, et al. Location of posterosuperior alveolar artery and correlation with maxillary sinus anatomy. Int $\mathrm{J}$ Periodontics Restorative Dent. 2015;35:e60-5.

26. Nedir R, Bischof M, VazquezL, Szmukler-Moncler S, Bernard JP. Osteotome sinus floor elevation without grafting material: a 1year prospective pilot study with ITI implants. Clin Oral Implants Res. 2006;17:679-86.
27.He L, Chang X, Liu Y. Sinus floor elevation using osteotome technique without grafting materials: a 2-year retrospective study. Clin Oral Implants Res. 2011;8:1-5.

28. Sohn DS, Moon JW, Lee WH, Kim SS, Kim CW, Kim KT, et al. Comparison of new bone formation in the maxillary sinus with and without bone grafts: immunochemical rabbit study. Int $\mathrm{J}$ Oral Maxillofac Implants. 2011;26:1033-42.

29. Hatano N, Sennerby L, Lundgren S. Maxillary sinus augmentation using sinus membrane elevation and peripheral venous blood for implant-supported rehabilitation of the atrophic posterior maxilla: Case series. Clin Implant DentRelat Res. 2007;9:150-5.

30. Sani E, Veltri M, Cagidiaco MC, Balleri P, Ferrari M. Sinus membrane elevation in combination with placement of blasted implants: a 3-year case report of sinus augmentation without grafting material. Int $\mathrm{J}$ Oral MaxillofacSurg. 2008;37:966-9.

31. Balleri P, Veltri M, Nuti N, Ferrari M. Implant placement in combination with sinus membrane elevation without Biomaterials: a 1-year study on 15patients. Clin Implant Dent Relat Res. 2012;14:682-9.

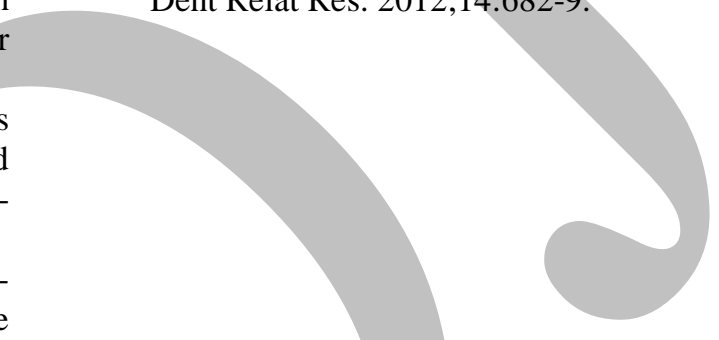

